\title{
An Australian risk prediction model for 30-day mortality after isolated coronary artery bypass: The AusSCORE
}

\author{
Christopher Reid, PhD, ${ }^{a}$ Baki Billah, PhD, ${ }^{\text {a }}$ Diem Dinh, PhD, ${ }^{\text {a }}$ Julian Smith, MBBS, ${ }^{\text {b }}$ \\ Peter Skillington, MBBS, ${ }^{\mathrm{c}}$ Michael Yii, MBBS, ${ }^{\mathrm{d}}$ Seven Seevanayagam, MBBS, \\ Morteza Mohajeri, MBBS, ${ }^{\mathrm{f}}$ and Gil Shardey, $\mathrm{MBBS}^{\mathrm{b}}$
}

\begin{abstract}
Objective: Our objective was to identify risk factors associated with 30-day mortality after isolated coronary artery bypass grafting in the Australian context and to develop a preoperative model for 30-day mortality risk prediction.

Summary Background Data: Preoperative risk associated with cardiac surgery can be ascertained through a variety of risk prediction models, none of which is specific to the Australian population. Recently, it was shown that the widely used EuroSCORE model validated poorly for an Australian cohort. Hence, a valid model is required to appropriately guide surgeons and patients in assessing preoperative risk.
\end{abstract}

\begin{abstract}
Methods: Data from the Australasian Society of Cardiac and Thoracic Surgeons database project was used. All patients undergoing isolated coronary artery bypass grafting between July 2001 and June 2005 were included for analysis. The data were divided into creation and validation sets. The data in the creation set was used to develop the model and then the model was validated in the validation set. Preoperative variables with a $P$ value of less than .25 in $\chi^{2}$ analysis were entered into multiple logistic regression analysis to develop a preoperative predictive model. Bootstrap and backward elimination methods were used to identify variables that are truly independent predictors of mortality, and 6 candidate models were identified. The Akaike Information Criteria (AIC) and prediction mean square error were used to select the final model (AusSCORE) from this group of candidate models. The AusSCORE model was then validated by average receiver operating characteristic, the $P$ value for the Hosmer-Lemeshow goodness-of-fit test, and prediction mean square error obtained from $n$-fold validation.

Results: Over the 4-year period, 11,823 patients underwent cardiac surgery, of whom 65.9\% (7709) had isolated coronary bypass procedures. The 30-day mortality rate for this group was $1.74 \%(134 / 7709)$. Factors selected as independent predictors in the preoperative isolated coronary bypass AusSCORE model were as follows: age, New York Heart Association class, ejection fraction estimate, urgency of procedure, previous cardiac surgery, hypercholesterolemia (lipid-lowering treatment), peripheral vascular disease, and cardiogenic shock. The average area under the receiver operating characteristic was 0.834 , the $P$ value for the Hosmer-Lemeshow $\chi^{2}$ test statistic was 0.2415 , and the prediction mean square error was 0.01869 .
\end{abstract}

Conclusion: We have developed a preoperative 30-day mortality risk prediction model for isolated coronary artery bypass grafting for the Australian cohort.

Risk models that predict mortality after isolated coronary artery bypass grafting $(\mathrm{CABG})$ have been developed in a variety of patient populations. Widely known models include the Parsonnet score (1989) ${ }^{1}$ and the European System for Cardiac Operative Risk Evaluation (EuroSCORE) model. ${ }^{2}$ The latter aimed to predict 30-day mortality in patients having cardiothoracic surgery. There are two Euro-

From CCRE Therapeutics, DEPM, Monash University, ${ }^{a}$ Melbourne, Australia; Department of Cardiothoracic Surgery, Monash Medical Centre, ${ }^{\mathrm{b}}$ Melbourne, Australia; Department of Cardiothoracic Surgery, Royal Melbourne Hospital, Melbourne, Australia; St Vincent's Hospital, ${ }^{\mathrm{d}}$ Melbourne, Australia; Department of Cardiothoracic Surgery, Austin Hospital, ${ }^{\mathrm{e}}$ Melbourne, Australia; and Department of Cardiothoracic Surgery, Geelong Hospital, ${ }^{\mathrm{f}}$ Victoria, Australia.

Received for publication Dec 13, 2007; revisions received Jan 18, 2009; accepted for publication March 8, 2009; available ahead of print June 1, 2009.

Address for reprints: Christopher Reid, PhD, CCRE Therapeutics, DEPM, Monash University, Alfred Hospital, Commercial Rd, Melbourne, VIC3004, Australia

(E-mail: chris.reid@med.monash.edu.au).

J Thorac Cardiovasc Surg 2009;138:904-10

$0022-5223 / \$ 36.00$

Copyright (c) 2009 by The American Association for Thoracic Surgery

doi:10.1016/j.jtcvs.2009.03.020
SCORE models with the same variables-the standard additive, ${ }^{2}$ which can be calculated by hand, and the multiple logistic, ${ }^{2}$ which is said to more accurately predict the risk in "high-risk" patients. The EuroSCORE has been validated in many populations, including The Society of Thoracic Surgeons database, ${ }^{2}$ Japan, ${ }^{3}$ and Cologne, Germany. ${ }^{4}$

In populations with no derived prediction models, three options can be used to estimate risk. ${ }^{5}$ These include (1) adopting a "ready made" model such as the Parsonnet or EuroSCORE, (2) "recalibrating" an existing model, using the risk factors from a published index and adjusting the weights so that it is relevant to the population, or (3) deriving a local index from the population's own surgical data set.

In Australia, the EuroSCORE model for risk adjustment is the most widely used. However, recently Yap and colleagues ${ }^{6}$ have shown that the EuroSCORE model could not be validated for use in the Australian population. Furthermore, Yap's group ${ }^{6}$ showed that recalibration of the EuroSCORE model did not provide an accurate model for the Australian 


\section{Abbreviations and Acronyms \\ AIC = Akaike Information Criteria \\ ASCTS $=$ Australian Society of Cardiac and Thoracic Surgeons \\ CABG = coronary artery bypass grafting \\ $\mathrm{CI}=$ confidence intervals \\ EuroSCORE $=$ European System for Cardiac Operative Risk Evaluation
MSE
NYHA
$=$ mean square error
ROC

cohort and identified the need for a new model to be developed that better reflected outcomes in the Australian population.

Therefore, the aim of this study was to develop a new model for predicting 30-day mortality after isolated CABG for use in the Australian population. Our hypothesis was that a locally derived model would provide a higher level of discrimination and would be better calibrated than the currently most widely used risk prediction model.

\section{METHODS \\ Database of the Australian Society of Cardiac and Thoracic Surgeons}

The Australian Society of Cardiac and Thoracic Surgeons (ASCTS) database has prospectively collected information about adult patients having cardiac surgery in 6 public hospitals in Victoria since June 2001. Each center has a data manager who is responsible for the completeness of the data collection. All data are verified on receipt, and data queries are generated from missing data, outliers, or inconsistencies reported. Data validation has been a major focus since the establishment of the ASCTS database in 2001. The data were subjected to both local validation and an external data quality audit program, which was performed on site to evaluate the completeness $(<1 \%$ missing data for any variable) and accuracy of the data housed within the combined database. The outcome from the audit was used to assist in further development of appropriate standards. ${ }^{7}$

The ASCTS database contains information on patient risk factors (including preoperative cardiac status and previous interventions), intraoperative details (including the procedure performed, myocardial protection, and procedural durations), and postoperative outcomes. All patients undergoing cardiac surgical procedures between July1, 2001, and June 30, 2005, were included in the database. The index outcome was mortality, defined as death within 30 days postoperatively. Thirty-day mortality information was obtained by data managers contacting patients, family members, or medical practitioners by telephone as part of clinical care.

\section{Statistical Methods}

All data were collected in a customized database, de-identified, and exported for data analysis. We have initially identified 20 variables as the risk factors for the 30-day mortality. Six of these variables contain missing observations. The New York Heart Association (NYHA) class has the highest rate of missing observations $(6.91 \%)$ followed by ejection fraction estimate $(0.51 \%)$ and body mass index $(0.18 \%)$. The variables creatinine history, previous myocardial infarction, and cerebrovascular disease each have less than $0.051 \%$ missing observations. Any patients with missing observations were excluded from the analysis. Stata version 9.2 and $\mathrm{R}$ version 2.3.1

were used for the analysis. ${ }^{8,9}$ The data have been randomly divided into model creation $(\mathrm{n}=5151,67 \%)$ and model validation sets $(\mathrm{n}=2558$, $33 \%$ ). The creation set was used to develop the model, and then the performance of the model was assessed in the validation set. The statistical significance for the independent association between a variable and 30-day mortality was assessed by the $\chi^{2}$ test. However, if the expected cell frequency for the 2-by-2 table was smaller than 5, Fisher's exact test was used for the significance test. The inclusion criterion for a variable in the $\chi^{2}$ analysis was that any of the observed frequency (prevalence) in the 2way table must not be less than $1 \%$ in the data set. After $\chi^{2}$ analysis, variables with a $P$ value of less than $25 \%$ were selected as plausible predictors into the multiple logistic regression model. We considered a $P$ value of .25 as the threshold value for ongoing multiple logistic regression analyses in accord with literature-based recommendations. ${ }^{10,11}$

Multiple logistic regression analysis and model development and testing were conducted by the bootstrap method. The bootstrap method was based on the random selection of 1000 repeated samples from the creation data set. The backward elimination method was used within each bootstrap sample to develop a parsimonious predictive model. ${ }^{10}$ In the variable selection process of backward elimination method, a $P$ value of .05 or less was regarded as significant. In all bootstrap samples, the number of times each candidate variable was identified as a significant predictive variable was recorded and then ranked. Six plausible multiple logistic regression models were developed on the basis of the proportion of times the variables were selected as significant predictors. The candidate models contained the variables that were identified as significant in at least $90 \%, 70 \%, 60 \%, 50 \%, 20 \%$, and $10 \%$ of the bootstrap samples.

The final model was selected using Akaike Information Criteria (AIC) and prediction mean square error (MSE) obtained for the creation data. ${ }^{12}$ The performance of the candidate models was compared by average receiver operating characteristic (ROC), Hosmer-Lemeshow ${ }^{11} P$ value, and prediction MSE calculated from $n$-fold $(n=50)$ validation in the validation data set. The first-degree interaction effect between clinically relevant risk factors and multicollinearity for all possible pairs of risk factors were also investigated (Figure 1).

Assignment of additive scores to risk factors was based on a linear transformation of the corresponding beta coefficient. The coefficient of each risk factor was divided by .6065 (absolute value of the lowest beta coefficient for hypercholesterolemia), multiplied by a constant of 2 , and rounded to the nearest integer. $^{13}$

\section{RESULTS}

A total of 11,823 procedures have been recorded in the ASCTS database from the commencement of recording, and of those $65.9 \%(n=7709)$ were isolated CABG procedures. Descriptive characteristics of these patients are shown in Table 1. The average age of the patients was $65.7 \pm 10.1$ years, and $76.8 \%$ of the patients were male. Elective surgery was performed in $58.9 \%$ of the patients, urgent surgery in $36.5 \%$, and emergency or salvage surgery in $4.52 \%$. Only $3.7 \%$ of patients had previous CABG surgery and $4.4 \%$ of patients had previous cardiac surgery.

The $\chi^{2}$ analysis revealed 20 candidate variables for the 30day mortality for CABG: age, gender, preoperative dialysis, creatinine level, respiratory disease, resuscitation less than 1 hour before the operation, use of inotropes, use of anticoagulants, use of nitrates, urgency of procedure, NYHA class, cerebrovascular disease, previous myocardial infarction, hypertension, cardiogenic shock, hypercholesterolemia (lipidlowering treatment), ejection fraction estimate, peripheral vascular disease, body mass index, and previous cardiac surgery. 

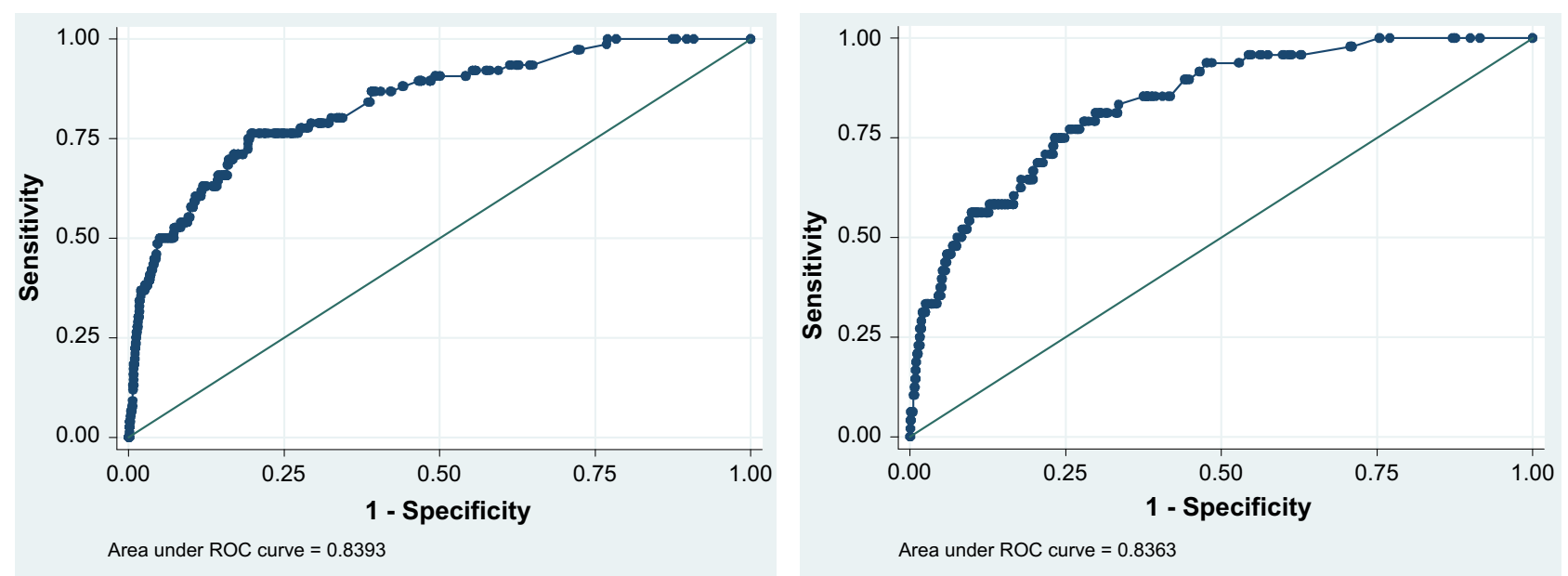

FIGURE 1. Single-validation receiver operating characteristic (ROC) for AusSCORE model for creation (left) and validation (right) data sets.

The results from bootstrap model selection are summarized in Table 2. No variable was identified as an independent predictor of mortality in all 1000 bootstrap samples. Five variables (age, NYHA class, ejection fraction estimate, urgency of procedure, and previous cardiac surgery) were identified in at least $90 \%$ of the bootstrap samples. Two additional variables (hypercholesterolemia and peripheral vascular disease) were selected in at least $70 \%$ of the samples. One variable (cardiogenic shock) was identified as an independent predictor in at least $60 \%$ of the samples. Inotrope medication was selected in at least $50 \%$ of bootstraps. The remaining 11 variables were selected as independent predictors of CABG mortality in less than $50 \%$ of the bootstrap samples.

We considered 6 plausible models on the basis of the proportion of times the variables were selected as significant in the bootstrap samples. For each model, the AIC and prediction MSE in the creation data set were obtained and are presented in Table 3. Clearly, the model with variables selected in at least $60 \%$ of the bootstrap samples gives the minimum AIC and MSE. Thus we consider this model as our final model, the AusSCORE. The risk factors in the AusSCORE are as follows: age, NYHA class, urgency of procedure, ejection fraction estimate, previous cardiac surgery, hypercholesterolemia (lipid-lowering treatment), peripheral vascular disease, and cardiogenic shock (Table 4). There was no evidence of multicollinearity or first-order interaction effect between the risk factors in the model. The beta coefficients, odds ratio, and the additive risk score are also presented in Table 4. The negative coefficient obtained for hypercholesterolemia may be driven by the lipid-lowering treatment. The average ROC, $P$ value, and MSE in the validation data set (n-fold validation), presented in Table 3, shows that our selected model had ROC $0.834, P$ value .2415 , and MSE 0.01869 . The model had the smallest prediction error, hence the best prediction performance among the candidate models.
For comparison with the EuroSCORE model, the ROC (0.8363) and $P$ value (.1477) were also calculated for the AusSCORE model using a single validation in the validation data set. The ROC and $P$ value for Hosmer-Lemeshow $\chi^{2}$ for goodness-of-fit test for the AusSCORE model for the Australian data and the EuroSCORE model for its own data and in the context of Australian data are presented in Table 5. The results suggest not only that the AusSCORE model calibrates well and is a valid model, but that it performs better than the EuroSCORE model. Actual versus predicted mortality

TABLE 1. Descriptive characteristics of patients undergoing isolated CABG

\begin{tabular}{lc}
\hline \multicolumn{1}{c}{ Characteristics } & $\begin{array}{c}\text { Descriptive measure } \\
(\mathbf{n}=\mathbf{7 7 0 9})\end{array}$ \\
\hline Age & $65.7 \pm 10.1$ \\
Body mass index & $28.2 \pm 4.6$ \\
Urgency of operation & \\
$\quad$ Elective & $58.9 \%$ \\
Urgent & $36.5 \%$ \\
Emergency & $4.2 \%$ \\
Salvage & $0.32 \%$ \\
Ejection fraction & \\
Normal & $45.3 \%$ \\
Mild & $31.3 \%$ \\
Moderate & $17.9 \%$ \\
Severe & $5.6 \%$ \\
Gender (male) & $76.8 \%$ \\
Hypercholeterolemia & $17.9 \%$ \\
Previous CABG & $3.7 \%$ \\
Previous surgery & $4.4 \%$ \\
Previous vascular disease & $13.6 \%$ \\
Cardiogenic shock & $1.6 \%$ \\
NYHA class & \\
Class I & $36.6 \%$ \\
Class II & $31.7 \%$ \\
Class III & $21.5 \%$ \\
Class IV & $10.2 \%$ \\
\hline CABG, Coronary artery bypass grafting; NYHA, New York Heart & $\mathrm{Association.}$ \\
&
\end{tabular}


TABLE 2. Number of times each candidate variable was selected in 1000 bootstrap samples drawn from the model creation dataset.

\begin{tabular}{lcc}
\hline \multicolumn{1}{c}{ Variable } & Frequency & $\%$ \\
\hline Age group & 982 & 98.2 \\
NYHA class & 955 & 95.5 \\
Urgency of procedures & 949 & 94.9 \\
Ejection fraction estimate & 948 & 94.8 \\
Previous cardiac surgery & 910 & 90.1 \\
Hypercholesterolemia & 787 & 78.7 \\
Peripheral vascular disease & 748 & 74.8 \\
Cardiogenic shock & 698 & 69.8 \\
Medications: inotropes & 558 & 55.8 \\
Hypertension & 495 & 49.5 \\
Gender & 476 & 47.6 \\
Body mass index group & 351 & 35.1 \\
Creatinine level $>0.133$ mmol/L & 337 & 33.7 \\
Preoperative dialysis & 323 & 32.3 \\
Resuscitation $<1$ hour preoperatively & 322 & 32.2 \\
Medications: nitrates & 252 & 25.2 \\
Medications: anticoagulants & 239 & 23.9 \\
Respiratory disease & 188 & 18.8 \\
Previous myocardial infarction & 185 & 18.5 \\
Cerebrovascular disease & 180 & 18.0 \\
\hline NYHA, New York Heart Association & &
\end{tabular}

for different risk subsets, presented in Table 6, also show that the AusSCORE model has a very good predicting performance. In the validation set, the overall observed mortality was $1.99 \%$ (95\% confidence intervals [CI] 1.45\%-2.53\%) and the predicted mortality was $1.53 \%$ (95\% CI $1.05 \%-$ $2.0 \%$ ). The low-risk group had an observed mortality of $0.20 \%(95 \%$ CI $0.0 \%-0.4 \%)$ and a predicted risk of $0.25 \%$ (95\% CI $0.0 \%-0.47 \%)$. The medium-risk group had an actual mortality of $1.04 \%$ (95\% CI $0.51 \%-1.57 \%$ ) and a predicted mortality of $0.7 \%$ (95\% CI $0.27 \%-$ $1.13 \%)$. The actual mortality for the high-risk group was $4.64 \%(95 \%$ CI $3.56 \%-5.72 \%)$ and the predicted mortality was $4.08 \%$ (95\% CI $3.06 \%-5.10 \%)$.

The estimated beta coefficients for the AusSCORE model reported in Table 4 can easily be used to predict the 30-day mortality risk for a patient undergoing cardiac surgery. Let us consider that a 75-year-old patient (beta coefficient is 1.2684) has the following 4 preoperative risk factors: NYHA class III (beta coefficient is .7383), urgent procedure type (beta coefficient is .8760), moderate ejection fraction estimate (beta coefficient is .7782), and peripheral vascular disease (beta coefficient is .7480). The constant term of the AusSCORE model is -6.2913 . The sum of the beta coefficients and the constant term is -1.8824 . Hence the predicted risk of 30-day mortality for this patient is as follows: predicted risk $=(\exp [-1.8824]) /(1+\exp [-1.8824])=0.1321$, or $13.21 \%(95 \%$ CI $3.4 \%-40 \%)$.

Additive risk score was also derived from the beta coefficients of the AusSCORE model (see Table 4) and additive risk categories were defined. The categories are as follows:
TABLE 3. Average ROC, Hosmer-Lemeshow $P$ value, and prediction MSE in an $n$-fold $(n=50)$ validation for the candidate models

\begin{tabular}{|c|c|c|c|c|c|}
\hline \multirow{2}{*}{$\begin{array}{l}\text { Percent of time } \\
\text { variables in the } \\
\text { bootstrap models }\end{array}$} & \multicolumn{2}{|c|}{ Creation data set } & \multicolumn{3}{|c|}{ Validation data set } \\
\hline & AIC & MSE & ROC & $\begin{array}{c}\text { H-L } \\
P \text { value } \\
\end{array}$ & MSE \\
\hline At least $90 \%$ & 668.3 & 0.01874 & 0.836 & .1323 & 0.01888 \\
\hline At least $70 \%$ & 660.7 & 0.01870 & 0.837 & .2730 & 0.01885 \\
\hline At least $60 \%$ & 657.8 & 0.01852 & 0.834 & .2415 & 0.01869 \\
\hline At least $50 \%$ & 657.8 & 0.01852 & 0.831 & .1153 & 0.01878 \\
\hline At least $20 \%$ & 661.9 & 0.01870 & 0.840 & .3130 & 0.01888 \\
\hline At least $10 \%$ (all) & 669.4 & 0.01871 & 0.838 & .1243 & 0.01890 \\
\hline
\end{tabular}

$R O C$, Receiver operating characteristic; $A I C$, Akaike Information Criteria; $M S E$, mean square error; $H-L$, Hosmer-Lemeshow.

less than 3, low risk (actual mortality rate $0.2 \%$ ); 3 to 5 , moderate risk (actual mortality rate 1.04\%); and above 5, high risk (actual mortality rate $4.64 \%$ ). The risk categories can easily be used to identify a patient's risk of 30-day mortality before the operation. A $\chi^{2}$ test showed that there is a strong association between mortality and risk categories $(P<.001)$, which demonstrates that the mortality rates in the high-risk categories are significantly higher than in the low-risk categories. The discrimination ability of the additive model (ROC 0.837 and 95\% CI 0.7840-0.899) was also very good and was similar to that of the logistic model (ROC 0.8363 and 95\% CI 0.7827-0.8898).

A validation of the AusSCORE model for all procedures data (isolated $\mathrm{CABG}$, valve[s] only, valve[s]+CABG, and others) showed that the global discrimination and calibration performances of the model were also very good $(\mathrm{ROC}=$ 0.8178 and Hosmer-Lemeshow $P$ value $=.5910$; overall actual mortality $=3.5 \%$, overall predicted mortality $=2.1 \%$ ). Thus the AusSCORE model can also be used to predict risk of 30-day mortality for the patients undergoing cardiac operations other than CABG as well.

\section{DISCUSSION}

This is the first article to provide a well-validated model for the prediction of 30-day mortality after isolated CABG surgery in an Australian population. It will allow for a better assessment of preoperative risk for both surgeons and patients to consider.

In the $\mathrm{CABG}$ risk model literature, the $\mathrm{ROC}$ for predictive models varies from 0.779 to $0.836 .^{14-19}$ Thus the performance of our proposed AusSCORE model with 8 variables (single validation: $\mathrm{ROC}=0.8363$ and $P$ value $=.1477$; n-fold validation: $\mathrm{ROC}=0.8340$ and $P$ value $=.2415)$ is comparable with the models reported in the literature and is better than the EuroSCORE model for its own data and especially for the Australian data. The need for a local risk model has been highlighted in the recent reports from Bidstrup, ${ }^{20}$ Yap $^{21}$ and their associates. In addition, Nashef ${ }^{22}$ (2006) recently flagged that one reason that the EuroSCORE 
TABLE 4. Beta coefficients (beta), odds ratio, and additive score for the preoperative isolated CABG AusSCORE model

\begin{tabular}{|c|c|c|c|c|c|}
\hline Risk factors & Beta & $P$ value & OR & $95 \%$ CI & Score \\
\hline \multicolumn{6}{|l|}{ Age group } \\
\hline$<60$ (reference) & & & 1.00 & & \\
\hline $60-70$ & .7487 & .091 & 2.11 & $(0.89,5.03)$ & 2 \\
\hline $70-80$ & 1.2684 & .002 & 3.56 & $(1.60,7.91)$ & 4 \\
\hline $80+$ & 1.7318 & .000 & 5.65 & $(2.13,14.98)$ & 6 \\
\hline \multicolumn{6}{|l|}{ NYHA class } \\
\hline Class I \& II (reference) & & & 1.00 & & \\
\hline Class III & .7383 & .016 & 2.09 & $(1.15,3.82)$ & 2 \\
\hline Class IV & 1.1998 & .000 & 3.12 & $(1.81,6.08)$ & 4 \\
\hline \multicolumn{6}{|l|}{ Urgency of procedure } \\
\hline Elective (reference) & & & 1.00 & & \\
\hline Urgent & .8760 & .003 & 2.40 & $(1.34,4.29)$ & 3 \\
\hline Emergency/salvage & 1.4226 & .012 & 4.15 & $(1.83,9.38)$ & 5 \\
\hline \multicolumn{6}{|l|}{ Ejection fraction } \\
\hline Normal/mild (reference) & & & 1.00 & & \\
\hline Moderate (30-45) & .7782 & .006 & 2.18 & $(1.25,3.81)$ & 3 \\
\hline Severe $(<30)$ & 1.2598 & .000 & 2.52 & $(1.86,6.68)$ & 4 \\
\hline Previous surgery & .8305 & .033 & 2.29 & $(1.07,4.92)$ & 3 \\
\hline Hypercholesterolemia & -.6065 & .021 & 0.55 & $(0.33,0.91)$ & -2 \\
\hline Peripheral vascular disease & .7480 & .005 & 2.11 & $(1.26,3.54)$ & 2 \\
\hline Cardiogenic shock & 1.0100 & .021 & 2.74 & $(1.16,6.47)$ & 3 \\
\hline Constant & -6.2913 & .000 & N/A & N/A & N/A \\
\hline
\end{tabular}

$C A B G$, Coronary artery bypass grafting; $O R$, odds ratio; $C I$, confidence intervals; $N Y H A$, New York Heart Association; $N / A$, not applicable. Score: Assignment of scores to risk factors was based on a linear transformation of the corresponding beta coefficient. The coefficient of each risk factor was divided by 0.6065 (absolute value of the lowest beta coefficient for hypercholesterolemia), multiplied by a constant of 2, and rounded to the nearest integer. Definitions: NYHA class I to IV: I: Patients with cardiac disease but without resulting limitation of physical activity. Ordinary physical activity does not cause undue fatigue, palpitations, or dyspnea. II: Patients with cardiac disease resulting in slight limitation of physical activity. They are comfortable at rest. Ordinary physical activity results in fatigue, palpitations, or dyspnea. III: Patients with cardiac disease resulting in marked limitation of physical activity. They are comfortable at rest. Less than ordinary physical activity results in fatigue, palpitations, or dyspnea. IV: Patients with cardiac disease resulting in inability to carry on any physical activity without discomfort. Symptoms of cardiac insufficiency may be present even at rest. If any physical activity is undertaken, discomfort is increased. Urgency of procedure: Not routine-medical reason for operating on this admission (1) within 72 hours from angiography if on the same admission that angiography was performed (in this case, "same admission" includes the situation when angiography is performed at another hospital and the patient is transferred directly to the hospital where surgery is to be performed) or (2) within 72 hours after an unplanned admission (in a patient who had a previous angiogram and was scheduled for surgery but was admitted acutely). Ejection fraction: The percentage of the blood emptied from the left ventricle at the end of the contraction. Use the most recent determination before intervention. Enter a percentage in the range of 5 to 90 . Previous CABG: Previous coronary artery bypass surgery by any approach. Hypercholesterolemia: Whether the patient has a history of hypercholesterolemia diagnosed and/or treated by a physician, and/or cholesterol level greater than $5.0 \mathrm{mmol}$. Peripheral vascular disease: Whether the patient has peripheral vascular disease, as indicated by claudication either with exertion or rest; amputation for arterial insufficiency; aortoiliac occlusive disease reconstruction; peripheral vascular bypass surgery, angioplasty, or stent; documented abdominal aortic aneurysm, abdominal aortic aneurysm repair, or stent; positive noninvasive testing documented. Cardiogenic shock: Is the patient, at the time of the procedure, in a clinical state of hypoperfusion according to either of the following criteria: (1) systolic blood pressure $\leq 80$ and/or cardiac index $\leq$ 1.8 despite maximal treatment; (2) intravenous inotropes and/or intra-aortic balloon pumping necessary to maintain systolic blood pressure $>80$ and/or cardiac index $>1.8$ ?

did not calibrate well to the Australian population was that the model is now over 10 years old and may not be appropriate for identifying risk in a contemporary cohort. The newly derived AusSCORE model will be valuable for providing preoperative 30-day mortality risk evaluation for surgeons and patients as well as providing a method for risk-adjusting surgical performance outcomes for quality assurance. The

TABLE 5. Comparison between AusSCORE and EuroSCORE models (single validation in the validation data set)

\begin{tabular}{|c|c|c|c|c|}
\hline \multirow[b]{2}{*}{ Model } & \multicolumn{2}{|c|}{ Validation } & \multicolumn{2}{|c|}{ Creation } \\
\hline & ROC & $\begin{array}{c}\text { H-L } \\
P \text { value }\end{array}$ & ROC & $\begin{array}{c}\text { H-L } \\
P \text { value }\end{array}$ \\
\hline AusSCORE & 0.8363 & .1477 & 0.8393 & .2871 \\
\hline EuroSCORE & 0.7590 & .6775 & 0.7875 & .4085 \\
\hline EuroSCORE in Australian data & 0.8200 & $<.05$ & N/A & N/A \\
\hline
\end{tabular}

$R O C$, Receiver operating characteristic; $H-L$, Hosmer-Lemeshow; $N / A$, not applicable.
AusSCORE model also performs well for the other surgical procedures (eg, valve[s] only, $\mathrm{CABG}+$ valve[s]). However, we plan to develop a separate risk prediction model for the Australian population using all procedures in a future study. We hope the new model will be more appropriate to predict 30-day mortality for global (all procedure types) cardiac surgery in Australia.

The methodologic approach of using bootstrap methods for developing predictive models for mortality was proposed to minimize the impact of automated variable selection methods. Automated selection methods have been shown to select noise variables as independent predictors, and these are likely to change on repeated (bootstrap) analyses. ${ }^{10}$ Hence, one should not rely solely on assessing the statistical significance of the predictors obtained by automated variable selection methods, because the related $P$ values are biased downward. ${ }^{23}$ In contrast, the bootstrap method can be used 
TABLE 6. Actual and AusSCORE predicted mortality and $95 \% \mathrm{CI}$ in the validation set

\begin{tabular}{lcc}
\hline \multicolumn{1}{c}{ Risk factors } & Actual (\%) & Predicted (\%) \\
\hline $\begin{array}{l}\text { Overall } \\
\text { Gender }\end{array}$ & $1.99(1.45-2.53)$ & $1.53(1.05-2.00)$ \\
$\quad$ Male & $2.63(1.32-3.94)$ & $1.73(0.66-2.80)$ \\
$\quad$ Female & $1.81(1.22-2.40)$ & $1.48(0.95-2.01)$ \\
Age group & & \\
$\quad<60$ & $0.97(0.25-1.70)$ & $0.62(0.05-1.19)$ \\
$60-70$ & $1.29(0.53-2.05)$ & $1.16(0.44-1.88)$ \\
$70-80$ & $2.98(1.83-4.13)$ & $2.23(1.23-3.23)$ \\
$80+$ & $5.37(1.75-9.00)$ & $4.09(0.91-7.27)$ \\
Urgency of procedure & & \\
$\quad$ Elective & $0.81(0.35-1.27)$ & $0.59(0.20-0.98)$ \\
$\quad$ Urgent & $2.52(1.52-3.52)$ & $2.10(1.19-3.01)$ \\
$\quad$ Emergency and salvage & $12.82(6.76-18.88)$ & $8.88(3.73-14.03)$ \\
NYHA class & & \\
Class I \& II & $1.35(0.79-1.91)$ & $0.74(0.32-1.16)$ \\
Class III & $2.44(1.13-3.75)$ & $2.05(0.85-3.25)$ \\
Class IV & $5.94(2.81-9.07)$ & $6.21(3.01-9.41)$ \\
Additive score & & \\
$\quad<3$ (low risk) & $0.20(0.00-0.40)$ & $0.25(0.00-0.47)$ \\
3-5 (medium risk) & $1.04(0.51-1.57)$ & $0.70(0.27-1.13)$ \\
$>5$ (high risk) & $4.64(3.56-5.72)$ & $4.08(3.06-5.10)$ \\
\hline CI Confden
\end{tabular}

CI, Confidence intervals; NYHA, New York Heart Association.

to assess the strength of evidence that a selected variable is indeed an independent predictor in the model. It is expected that the truly independent predictors would be selected in the majority of the bootstrap samples; however, noise variables would be identified as independent predictors in a minority of samples. The methods adopted in the present analyses have provided a stable AusSCORE model that has been shown to perform better than the current conventional risk prediction models. Five risk factors-age, urgency of operation, ejection fraction estimate, previous cardiac surgery, and peripheral vascular disease-appeared in both EuroSCORE and AusSCORE models. The absence of other EuroSCORE variables in the AusSCORE may be related to their association with non-CABG surgical outcomes.

A limitation of the current study may be the relatively limited number of cases included in the ASCTS database. However, with the 6 public hospitals contributing to the data set, the analysis was undertaken after 4 years of collection, and one may argue that further delay may result in the lack of a contemporary data set for development. In addition, the data were collected in one Australian state and not across all states. This will be avoided through expansion of the ASCTS database to include surgical units across Australia, and we would propose a review of the AusSCORE after the compilation of 20,000 cases.

\section{CONCLUSIONS}

We have developed an 8-variable AusSCORE model for predicting 30-day mortality for isolated $\mathrm{CABG}$ procedures in Australia. The strength of the AusSCORE model compared with similar models in the literature is the use of bootstrap methods for variable selection. This provides a highly consistent set of predictor variables associated with 30-day mortality after isolated CABG surgery. The performance of the AusSCORE model is found to be better than that of the EuroSCORE model for its own data and its application to the Australian data. All of the risk factors identified in the AusSCORE model are generally considered as risk factors by most cardiac surgeons and cardiologists. However, a risk prediction model based on the Australian data was overdue inasmuch as the EuroSCORE model did not calibrate well for the Australian data. We recommend the use of the AusSCORE model for the Australian cohort.

\section{References}

1. Parsonnet V, Dean D, Bernstein AD. A method of uniform stratification of risk for evaluating the results of surgery in acquired adult heart disease. Circulation 1989;79(Suppl 1):I-3-I12.

2. Nashef SA, Roques F, Hammill BG, Peterson ED, Michel P, Grover FL, et al. Validation of European System for Cardiac Operative Risk Evaluation (EuroSCORE) in North American cardiac surgery [see comment]. Eur J Cardiothorac Surg. 2002;22:101-5.

3. Kawachi Y, Nakashima A, Toshima Y, Arinaga K, Kawano H. Risk stratification analysis of operative mortality in heart and thoracic aorta surgery: comparison between Parsonnet and EuroSCORE additive model. Eur J Cardiothorac Surg. 2001;20:961-6.

4. Geissler HJ, Hölzl P, Marohl S, Kuhn-Régnier F, Mehlhorn U, Südkamp M, et al. Risk stratification in heart surgery: comparison of six score systems. Eur J Cardiothorac Surg. 2000; 17:400-6.

5. Ivanov J, Tu JV, Naylor CD. Ready-made, recalibrated, or remodeled?: Issues in the use of risk indexes for assessing mortality after coronary artery bypass graft surgery. Circulation. 1999;99:2098-104

6. Yap C-H, Reid C, Yii M, Rowland MA, Mohajeri M, Skillington PD, et al. Validation of the EuroSCORE model in Australia. Eur J Cardiothorac Surg. 2006; 29:441-6.

7. Reid CM, Rockell M, Skillington PD, Shardey GC, Smith JA, Yii M, et al. Initial twelve months experience and analysis for 2001-2002 from the Australasian Society of Cardiac and Thoracic Surgeons-Victorian database project. Heart Lung Circ. 2004;13:291-7.

8. StataCorp. Stata Statistical Software, version 9.2 [computer software]. College Station (TX): Stata Corporation; 2004.

9. R Development Core Team. R: A language and environment for statistical computing 2.3.1.: ed. Vienna, Austria: R Foundation for Statistical Computing; 2006.

10. Austin P, Tu J. Bootstrap methods for developing predictive models. Am Stat 2004;58:131-7.

11. Hosmer DW, Lemeshow S. Applied logistic regression. New York: John Wiley \& Sons; 1989.

12. Akaike H. Statistical predictor identification. Ann Inst Math Stat. 1970;22:203-17.

13. Rassi A Jr, Rassi A, Little WC, Xavier SS, Rassi SG, Rassi AG, et al. Development and validation of a risk score for predicting death in Chagas' heart disease. N Engl J Med. 2006;355:799-808.

14. Morrow DA, Antman EM, Charlesworth A, Cairns R, Murphy SA, de Lemos JA, et al. TIMI risk score for ST-elevation myocardial infarction: a convenient, bedside, clinical score for risk assessment at presentation: an intravenous nPA for treatment of infarcting myocardium early II trial substudy. Circulation. 2000; 102:2031-7.

15. Morrow DA, Antman EM, Parsons L, de Lemos JA, Cannon CP, Giugliano RP, et al. Application of the TIMI risk score for ST-elevation MI in the National Registry of Myocardial Infarction 3. JAMA. 2001;286:1356-9.

16. Boersma E, Pieper KS, Steyerberg EW, Wilcox RG, Chang WC, Lee KL, et al Predictors of outcome in patients with acute coronary syndromes without persistent ST-segment elevation. Results from an international trial of 9461 patients. The PURSUIT Investigators. Circulation. 2000;101:2557-67.

17. Jacobs AK, Kelsey SF, Brooks MM, Faxon DP, Chaitman BR, Bittner V, et al Better outcome for women compared with men undergoing coronary 
revascularization: a report from the bypass angioplasty revascularization investigation (BARI). Circulation. 1998;98:1279-85.

18. Krumholz HM. Mathematical models and the assessment of performance in cardiology. Circulation. 1999;99:2067-9.

19. Lee KL, Woodlief LH, Topol EJ, Weaver WD, Betriu A, Col J, et al. Predictors of 30-day mortality in the era of reperfusion for acute myocardial infarction. Results from an international trial of 41,021 patients. GUSTO-I Investigators. Circulation. 1995;91:1659-68.
20. Bidstrup B. Towards an Australian national cardiac surgery database. ANZ J Surg. 2005;75:505-6.

21. Yap C-H, Mohajeri M, Ihle BU, Wilson AC, Goyal S, Yii M. Validation of EuroSCORE model in an Australian patient population. ANZ J Surg. 2005; 75:508-12.

22. Nashef S. Editorial comment. Eur J Cardiothoracic Surg. 2006;29:446.

23. Harrell WA, Spaulding LM. Social psychological models of choice behavior and drivers' left turns. J Soc Psychol. 2001;141:714-22. 